\title{
An acceleration of convergence to some generalized-Euler-constant function
}

\author{
V. Lampret
}




\title{
AN ACCELERATION OF CONVERGENCE TO SOME GENERALIZED-EULER-CONSTANT FUNCTION
}

\author{
V. LAMPRET
}

Received 28 June, 2012

Abstract. For the generalized-Euler-constant function $\gamma(a)$,

$$
\gamma(a):=\lim _{n \rightarrow \infty}\left(\sum_{k=0}^{n-1} \frac{1}{a+k}-\ln \frac{a+n-1}{a}\right) \quad(a>0),
$$

and for any positive integer $q \geq 2$, using the Bernoulli numbers $B_{2 m}$, the sequences $n \mapsto$ $\mathfrak{A}_{n}(a, q), n \mapsto \mathfrak{B}_{n}(a, q)$ and $n \mapsto \mathfrak{C}_{n}(a, q)$, having the properties

$$
\begin{aligned}
& \lim _{n \rightarrow \infty} n^{2 q-2}\left[\gamma(a)-\mathfrak{A}_{n}(a, q)\right]=\frac{B_{2 q-2}}{2 q-2}, \\
& \lim _{n \rightarrow \infty} n^{2 q-2}\left[\gamma(a)-\mathfrak{B}_{n}(a, q)\right]=-\left(1-2^{3-2 q}\right) \frac{B_{2 q-2}}{2 q-2}
\end{aligned}
$$

and

are determined.

$$
\lim _{n \rightarrow \infty} n^{2 q-1}\left[\gamma(a)-\mathfrak{C}_{n}(a, q)\right]=\frac{1}{2} B_{2 q-2},
$$

2010 Mathematics Subject Classification: 11B83; 40A05; 11B68; 33E20

Keywords: acceleration of convergence, generalized Euler's constant, generalized $\gamma$-sequence

\section{INTRODUCTION}

The gamma-sequence

$$
y_{n}(a)=\sum_{k=0}^{n-1} \frac{1}{a+k}-\ln \frac{a+n-1}{a} \quad(n \in \mathbb{N}),
$$

considered in [2,3] is convergent for $a>0$ and defines the generalized-Euler-constant function $\gamma(a)$,

$$
\gamma(a):=\lim _{n \rightarrow \infty} y_{n}(a)
$$


The name generalized-Euler-constant function has its origin in the identity $\gamma(1)=$ $C$, where $C$ is the Euler-Mascheroni constant. Several results on the rate of convergence of the sequence (1.1) have been established in the literature.

Recently, A. Sîntămărian [4] accelerated the convergence (1.2) using the StolzCesaro limit theorem. In this reference the sequences

$$
\begin{aligned}
\alpha_{n, 2}(a) & :=\sum_{k=0}^{n-1} \frac{1}{a+k}-\frac{1}{2(a+n-1)}+\frac{1}{12(a+n-1)^{2}} \\
& -\ln \left(\frac{a+n-1}{a}+\frac{1}{120 a(a+n-1)^{3}}\right)
\end{aligned}
$$

and

$$
\beta_{n, 2}(a):=\alpha_{n, 2}(a)+\frac{1}{252(a+n-1)^{6}}
$$

were considered and in Theorem 2 the equalities

$$
\lim _{n \rightarrow \infty} n^{6}\left[\gamma(a)-\alpha_{n, 2}(a)\right]=\frac{1}{252}
$$

and

$$
\lim _{n \rightarrow \infty} n^{8}\left[\beta_{n, 2}(a)-\gamma(a)\right]=\frac{121}{28800}
$$

were derived. Similarly, in Theorem 3, were considered some sequences $\alpha_{n, 3}(a)$, $\beta_{n, 3}(a)$ and $\delta_{n, 3}(a)$ such that the following limits hold:

$$
\begin{aligned}
& \lim _{n \rightarrow \infty} n^{8}\left[\alpha_{n, 3}(a)-\gamma(a)\right]=\frac{1}{240}, \\
& \lim _{n \rightarrow \infty} n^{10}\left[\gamma(a)-\beta_{n, 3}(a)\right]=\frac{1}{132}
\end{aligned}
$$

and

$$
\lim _{n \rightarrow \infty} n^{12}\left[\delta_{n, 3}(a)-\gamma(a)\right]=\frac{174197}{8255520} .
$$

In [4] the equalities above were demonstrated using rather tedious calculations.

The goal of this article is to complement/improve the results and the method of derivation as presented in [4]. In our paper we present an approach of incessant acceleration of the convergence (1.1) to any degree. We will present three classes of sequences converging to $\gamma(a)$ much faster than the original sequence $y_{n}(a)$ does. 


\section{PRELIMINARIES}

Referring to (1.1), (1.2) and [1, Theorems 1-3], we have the following equalities ${ }^{1}$

$$
\begin{aligned}
\gamma(a) & =S_{n}(a, q)+R_{n}(a, q) & & (n, q \in \mathbb{N}) \\
& =\sigma_{n}(a, q)+\rho_{n}(a, q) & & (n, q \in \mathbb{N}) \\
& =S_{n}^{*}(a, q)+R_{n}^{*}(a, q) & & (n, q \in \mathbb{N})
\end{aligned}
$$

with $^{2}$

$$
\begin{aligned}
& S_{n}(a, q)=\sum_{k=0}^{n-1} \frac{1}{a+k}-\ln \frac{a+n}{a}+\frac{1}{2(a+n)}+\sum_{j=1}^{q-1} \frac{B_{2 j}}{2 j(a+n)^{2 j}}, \\
& \sigma_{n}(a, q)=\sum_{k=0}^{n-1} \frac{1}{a+k}+\ln \left(\frac{a}{a+n-\frac{1}{2}}\right)-\sum_{i=1}^{q-1}\left(\frac{1-2^{1-2 i}}{2 i} \cdot \frac{B_{2 i}}{\left(a+n-\frac{1}{2}\right)^{2 i}}\right)
\end{aligned}
$$

and

$$
\begin{aligned}
S_{n}^{*}(a, q) & =\sum_{k=0}^{n-1} \frac{1}{a+k}-\ln \frac{a+n}{a} \\
& -1-\ln \left(1-\frac{1}{a+n+1}\right)^{a+n+1}+\frac{1}{2(a+n)}-\frac{1}{2} \ln \left(1+\frac{1}{a+n}\right) \\
& -\sum_{j=1}^{q-1} \frac{B_{2 j}}{(2 j)(2 j-1)}\left[\frac{1}{(a+n)^{2 j-1}}-\frac{1}{(a+n+1)^{2 j-1}}-\frac{2 j-1}{(a+n)^{2 j}}\right] .
\end{aligned}
$$

The remainders are estimated as

$$
\begin{aligned}
\left|R_{n}(a, q)\right| & <\frac{\left|B_{2 q}\right|}{q(a+n)^{2 q}}, \\
\left|\rho_{n}(a, q)\right| & <\frac{\left|B_{2 q}\right|}{q\left(a+n-\frac{1}{2}\right)^{2 q}}
\end{aligned}
$$

\footnotetext{
${ }^{1}$ The sequence $\sigma_{n}(a, q)$ in the expression (2.5) is given in the corrected form appearing in the proof of [1, Theorem 2], where in the first sum the start " $k=1$ " should be replaced by " $k=0$ " and where the summands in the third sum of $\sigma_{n}(a, q)$ are written incorrectly.

${ }^{2}$ By definition $\sum_{k=1}^{m} x_{k}=0$ for $m<1$.
} 
and

$$
\left|R_{n}^{*}(a, q)\right|<\frac{\left|B_{2 q}\right|}{(a+n)^{2 q+1}} .
$$

Here, the symbol $B_{k}$ means the $k$-th Bernoulli number,

$$
\frac{t e^{x t}}{e^{t}-1} \equiv \sum_{k=0}^{\infty} B_{k}(x) \frac{t^{j}}{j !} \quad(x \in \mathbb{R},|t|<2 \pi),
$$

$B_{k} \equiv B_{k}(0), B_{k}(x)$ is $k$-th Bernoulli polynomial.

\section{AN ACCELERATION OF CONVERGENCE}

Referring to (2.4)-(2.6) we make the following definition.

Definition 1. For any $a>0$ and any integer $q \geq 2$ we consider the following sequences:

$$
\begin{aligned}
n & \mapsto \mathfrak{A}_{n}(a, q):=S_{n}(a, q-1), \\
n & \mapsto \mathfrak{B}_{n}(a, q):=\sigma_{n}(a, q-1)
\end{aligned}
$$

and

$$
n \mapsto \mathfrak{C}_{n}(a, q):=S_{n}^{*}(a, q-1) .
$$

Now, we are in the position to formulate the following result.

Theorem 1. For any positive $a$ and any integer $q \geq 2$ we have the following limits:

$$
\begin{array}{ll}
\lim _{n \rightarrow \infty} n^{2 q-2}\left[\gamma(a)-\mathfrak{A}_{n}(a, q)\right]=\frac{B_{2 q-2}}{2 q-2} & =: L_{\mathfrak{A}}(q), \\
\lim _{n \rightarrow \infty} n^{2 q-2}\left[\gamma(a)-\mathfrak{B}_{n}(a, q)\right]=-\left(1-2^{3-2 q}\right) \frac{B_{2 q-2}}{2 q-2} & =: L_{\mathfrak{B}}(q)
\end{array}
$$

and

$$
\lim _{n \rightarrow \infty} n^{2 q-1}\left[\gamma(a)-\mathfrak{C}_{n}(a, q)\right]=\frac{1}{2} B_{2 q-2} \quad=: L_{\mathfrak{C}}(q) .
$$

Note that the limits are independent of $a$.

Proof. According to (2.1), (2.4) and (3.1), we have

$$
\gamma(a)=\mathfrak{A}_{n}(a, q)+\frac{B_{2 q-2}}{(2 q-2)(a+n)^{2 q-2}}+R_{n}(a, q),
$$


for $n \geq 1, a>0$ and $q \geq 2$. Consequently, using (2.7), the equality (3.4) follows. Similarly, referring to (2.2), (2.5) and (3.2), we get

$$
\gamma(a)=\mathfrak{B}_{n}(a, q)-\frac{1-2^{3-2 q}}{2 q-2} \cdot \frac{B_{2 q-2}}{\left(a+n-\frac{1}{2}\right)^{2 q-2}}+\rho_{n}(a, q),
$$

for $n \geq 1, a>0$ and $q \geq 2$. Thus, considering (2.8), we confirm (3.5). Finally, referring to (2.3), (2.6) and (3.3) we obtain

$$
\begin{aligned}
\gamma(a)= & \mathfrak{C}_{n}(a, q)+R_{n}^{*}(a, q) \\
& +\frac{B_{2 q-2}}{(2 q-2)(2 q-3)}\left[\frac{1}{(a+n+1)^{2 q-3}}-\frac{1}{(a+n)^{2 q-3}}+\frac{2 q-3}{(a+n)^{2 q-2}}\right],
\end{aligned}
$$

for $n \geq 1, a>0$ and $q \geq 2$. Denoting $a+n=b, 2 q-3=m$ and using Taylor's formula of order 1 around $b$ for the function $f(x) \equiv x^{-m},(b+1)^{-m}=b^{-m}$ $m b^{-(m+1)}+\frac{1}{2} m(m+1)(b+\vartheta)^{-(m+2)}$, we obtain the equality

$$
\frac{1}{(a+n+1)^{2 q-3}}=\frac{1}{(a+n)^{2 q-3}}-\frac{2 q-3}{(a+n)^{2 q-2}}+\frac{(2 q-3)(2 q-2)}{2(a+n+\vartheta)^{2 q-1}},
$$

for some $\vartheta=\vartheta_{n}(a, q) \in(0,1)$. From (3.7) and (3.8) we get the expression

$$
\gamma(a)-\mathfrak{C}_{n}(a, q)=\frac{B_{2 q-2}}{(2 q-2)(2 q-3)} \cdot \frac{(2 q-3)(2 q-2)}{2(a+n+\vartheta)^{2 q-1}}+R_{n}^{*}(a, q),
$$

which, recalling (2.9), demonstrates the relation (3.6).

Example 1. Referring to (3.4)-(3.6) and using [5] we obtain the following tables:

\begin{tabular}{|c||c|c|c|c|c|c|}
\hline$q$ & 2 & 3 & 4 & 5 & 6 & 7 \\
\hline$L_{\mathfrak{A}}(q)$ & $\frac{1}{12}$ & $-\frac{1}{120}$ & $\frac{1}{252}$ & $-\frac{1}{240}$ & $\frac{1}{132}$ & $-\frac{691}{32760}$ \\
\hline
\end{tabular}

TABLE 1. The type $\mathfrak{A}$-limits; Theorem 1, Eq. (3.4).

\begin{tabular}{|c||c|c|c|c|c|c|}
\hline$q$ & 2 & 3 & 4 & 5 & 6 & 7 \\
\hline$L_{\mathfrak{B}}(q)$ & $-\frac{1}{24}$ & $\frac{7}{960}$ & $-\frac{31}{8064}$ & $\frac{127}{30720}$ & $-\frac{511}{67584}$ & $\frac{1414477}{67092480}$ \\
\hline
\end{tabular}

TABlE 2. The type $\mathfrak{B}$-limits; Theorem 1, Eq. (3.5). 


\begin{tabular}{|c||c|c|c|c|c|c|}
\hline$q$ & 2 & 3 & 4 & 5 & 6 & 7 \\
\hline$L_{\mathfrak{C}}(q)$ & $\frac{1}{12}$ & $-\frac{1}{60}$ & $\frac{1}{84}$ & $-\frac{1}{60}$ & $-\frac{5}{132}$ & $-\frac{691}{5460}$ \\
\hline
\end{tabular}

TABLE 3. The type $\mathfrak{C}$-limits; Theorem 1, Eq. (3.6).

\section{ACKNOWLEDGEMENT}

The author express sincere thanks to the referee whose comments resulted in a significant improvement of the paper.

\section{REFERENCES}

[1] V. Lampret, "A double inequality for a generalized-Euler-constant function," J. Math. Anal. Appl., vol. 381, no. 1, pp. 155-165, 2011.

[2] A. Sîntamarian, "Some inequalities regarding a generalization of Euler's constant," JIPAM, J. Inequal. Pure Appl. Math., vol. 9, no. 2, p. 7, 2008.

[3] A. Sîntămărian, “A generalization of Euler's constant,” Numer. Algorithms, vol. 46, no. 2, pp. 141$151,2007$.

[4] A. Sîntămărian, "New sequences that converge to a generalization of Euler's constant," Integers, vol. 11, no. 2, pp. 127-138, A11, 2011.

[5] S. Wolfram, Mathematica, version 7.0. Wolfram Research, Inc., 1988-2009.

Author's address

\section{Lampret}

University of Ljubljana, Faculty of Civil and Geodetic Engineering, Jamova 2, 1000 Ljubljana, Slovenia

E-mail address: vito. lampret@fgg.uni-lj.si 